\title{
Negative impact of occupational exposure on surgical outcome in patients with rhinosinusitis
}

\author{
V. Hox ${ }^{1,2, *}$, S. Delrue ${ }^{1, *}$, H. Scheers ${ }^{2}$, E. Adams $^{3}$, S. Keirsbilck ${ }^{3}$, M. Jorissen ${ }^{1}$, P. H. Hoet ${ }^{2}$, \\ J. A. Vanoirbeek ${ }^{2}$, B. Nemery ${ }^{2,3} \&$ P. W. Hellings ${ }^{1}$ \\ ${ }^{1}$ Department of Otorhinolaryngology, Head and Neck Surgery, University Hospitals Leuven, Leuven, Belgium; ${ }^{2}$ Department of Public Health, \\ Research Unit of Lung Toxicology, University of Leuven, Leuven, Belgium; ${ }^{3}$ Division of Pneumology, Clinic of Occupational and \\ Environmental Medicine, University Hospitals Leuven, Leuven, Belgium
}

To cite this article: Hox V, Delrue S, Scheers H, Adams E, Keirsbilck S, Jorissen M, Hoet PH, Vanoirbeek JA, Nemery B, Hellings PW. Negative impact of occupational exposure on surgical outcome in patients with rhinosinusitis. Allergy 2012; DOI:10.1111/1.1398-9995.2011.0.2779.x

\section{Keywords}

occupational medicine; sinusitis

\section{Correspondence}

Peter W. Hellings, MD, PhD, Department of Otorhinolaryngology, Head and Neck Surgery, University Hospitals Leuven, Kapucijnenvoer 33, 3000 Leuven, Belgium.

Tel.: 003216332338

Fax: 003216346035

E-mail: peter.hellings@med.kuleuven.be

*These authors contributed equally.

Accepted for publication 01 December 2011

DOI:10.1111/j.1398-9995.2011.02779.x

Edited by: Wytske Fokkens

\begin{abstract}
Background: Chronic rhinosinusitis (CRS) is a frequent condition that is treated by functional endoscopic sinus surgery (FESS) when medical treatment fails. Endogenous as well as exogenous factors may be responsible for persisting symptoms after FESS. The role of occupational exposures on success of FESS has never been investigated.

Methods: In this case-control study, we tested the hypothesis that the outcome of FESS procedures is related to exposures at work. Questionnaires were sent to 890 patients who had undergone one or more FESS procedures and to 182 controls. Three independent experts assessed blindly the reported work exposures to inhaled agents. The relationship between exposure and the number of FESS procedures was analyzed.

Results: Relevant occupational exposure was reported by $25 \%$ of all responding patients undergoing FESS $(n=467)$ and $12 \%$ of controls $(n=69)$. The prevalence of occupational exposures increased linearly with the number of FESS procedures from $21 \%$ in those who had one FESS to $44 \%$ in those who had four or more FESS $\left(\chi^{2}=12.74, P<0.001\right)$. Logistic regression analysis with adjustments for potential confounders, including smoking, atopy, and asthma, confirmed that the odds ratio (OR) for reporting occupational exposures was significantly higher in those needing more than one FESS $(\mathrm{OR}=1.64)$ or more than two FESS $(\mathrm{OR}=1.97)$. These results were mainly driven by exposure to low molecular weight agents.

Conclusion: Exposure at work appears to be a risk factor for the occurrence of CRS and for its recurrence or persistence, as evidenced by the need for revision surgery.
\end{abstract}

Acute and chronic rhinosinusitis (ARS and CRS) are common diseases, affecting up to $10 \%$ of the Western population (1). Allergy is considered to be a risk factor for the development of rhinosinusitis (1-3), but several other immunologic, microbiologic, and genetic factors contribute to the complex pathophysiology of sinonasal inflammation $(1,4-7)$. Nowadays, good medical treatment options are available for patients affected by rhinosinusitis (1). In those patients with insufficient symptom control, functional endoscopic sinus surgery (FESS) is the treatment of choice. However, about $10-15 \%$ of operated patients respond insufficiently to FESS, and revision surgery is necessary (1). Known predictors for revision surgery are extensive sinonasal polyps, bronchial asthma, aspirin intolerance, and cystic fibrosis (8-11). Data on the role of atopy on FESS outcome are contradictory $(12,13)$. Clinical experience, however, points toward a higher occurrence of FESS failure in patients who are occupationally exposed to agents that may interfere with the postoperative healing. To our knowledge, the link between occupational factors and failure of FESS has not been investigated so far.

Occupational agents can be classified as high molecular weight (HMW) compounds ( $>5 \mathrm{kDa})$ or low molecular weight (LMW) compounds $(<5 \mathrm{kDa})$. The LMW compounds are again subdivided into two groups, depending on 
their sensitization capacity; LMW sensitizers, such as isocyanates, persulphate salts, and acid anhydrides, lead to allergic inflammation after immunologic sensitization (14), whereas LMW irritants, such as chlorine, ammonia, or ozone, cause airway injury and inflammation through nonallergic pathways (14).

The impact of occupational agents on the incidence and clinical presentation of sinus disease is unknown. Here, we present a large-scale retrospective study in which we assessed the self-reported exposure to occupational agents in patients who had undergone one or more FESS for rhinosinusitis. We tested the hypothesis that rhinosinusitis and especially the number of FESS procedures are related to exposures at work.

\section{Materials and methods}

\section{Study population}

A total of 890 patients who underwent a FESS between January 2004 and December 2008 in the Department of Ear, Nose and Throat Diseases of the University Hospitals of Leuven were enrolled. All patients were between 18 and 65 years of age. Indications for performing FESS included recurrent ARS and CRS with or without nasal polyps. Patients undergoing FESS for other reasons such as sinusitis of dental origin, fungus balls, structural lesions, and benign and malignant neoplasms, or who suffered from diseases such as primary ciliary dyskinesia, cystic fibrosis, or known immune deficiencies, were excluded from this study. FESS was performed either unilaterally or bilaterally, and the extent of surgery accorded to internationally accepted guidelines (1). Standard postoperative care consisted of nasal saline irrigation, oral corticosteroids, and weekly suction cleaning during the first 3 weeks.

In addition to the patients with rhinosinusitis, we also included a control group consisting of 182 subjects without a history of FESS, who had undergone vocal cord surgery for benign structural lesions. We chose this control group mainly because of a similar age distribution to that of the FESS population.

Atopy was based on medical records, as all patients had a blood analysis for specific IgE or skin prick tests for the most common inhalant allergens. Asthma was based on selfreporting.

The study was approved by the local ethics committee of the University Hospitals of Leuven.

\section{Questionnaire}

An extensive questionnaire based on and modified from 'the occupational history form' proposed by Bernstein et al. (15) was sent by mail to the study population. Patients were asked about their job at the time of the last sinus surgery. Questions on exposure included occupational and recreational exposures, duration of exposures, type of agents (including an extensive list to choose from), and sinonasal symptoms related to exposures. In addition, general questions about smoking, medication use, comorbidities, current sinonasal symptoms, presence of self-reported asthma, and occupational history were included.

\section{Analysis of questionnaire responses}

All returned questionnaires were analyzed independently and blindly by three physicians specialized in occupational medicine (V.H., E.A., and S.K.). Exposure at the time of last FESS was judged as 'relevant' only when two of three experts scored it as positive for occupational exposure. Occupational agents were categorized as being HMW agents, LMW sensitizers, or irritants.

\section{Additional telephone survey}

After the analysis of returned questionnaires, we contacted a random 110 subjects (including cases and controls, responders, and nonresponders) by telephone to assess possible response biases, as well as the validity of the responses to the postal questionnaire. All questions from the original mailed questionnaire were repeated and completed.

\section{Statistical analysis}

Statistical analyses were performed in SAS software, version 9.2 (SAS Institute, Cary, NC, USA), and SPSS, version 17.0 (SPSS Inc., Chicago, IL, USA). Logistic regression analysis was used to calculate the odds of having a relevant exposure in subjects having undergone one or more FESS, compared to control subjects. A proportional odds model (POM), which allows more than two ordered response categories, and a Poisson regression model with log link, handling the number of FESS as count data, were used to analyze the relationship between an increasing number of FESS and exposure state. Possible confounding by asthma, current smoking state, presence of nasal polyps, and atopy was investigated by three-way analysis of contingency tables and by examining their relationship with both predictor and response variables in the POM and Poisson regression model. All tests were two-sided with $\alpha=0.05$, and results are presented as odds ratios (OR) with 95\% confidence intervals $(95 \% \mathrm{CI})$.

\section{Results}

\section{Patient characteristics}

Of the 890 patients undergoing FESS, 467 returned a filled questionnaire $(52.5 \%$ response rate), compared with 69 of 182 control subjects $(37.9 \%$ response rate). Of the patients undergoing FESS that responded, 59.5\% had undergone one surgery $(n=278), 25.7 \%$ two surgeries $(n=120), 9.0 \%$ three surgeries $(n=42)$, and $5.8 \%(n=27)$ had undergone four or more surgeries. About $27 \%$ of the patients undergoing FESS were diagnosed with nasal polyps $(n=126)$. General patient characteristics are listed in Table 1. Active smoking, atopy, and self-reported asthma were more 
Table 1 Patient characteristics

\begin{tabular}{|c|c|c|c|c|c|c|}
\hline & $\begin{array}{l}\text { Controls } \\
(n=69)\end{array}$ & $\begin{array}{l}1 \text { FESS } \\
(n=278)\end{array}$ & $\begin{array}{l}2 \text { FESS } \\
(n=120)\end{array}$ & $\begin{array}{l}3 \text { FESS } \\
(n=42)\end{array}$ & $\begin{array}{l}+3 \text { FESS } \\
(n=27)\end{array}$ & $P$-value \\
\hline \multicolumn{7}{|l|}{ Age (years) } \\
\hline Mean $( \pm S D)$ & $46( \pm 13)$ & $47( \pm 12)$ & $47( \pm 11)$ & $47( \pm 12)$ & $47( \pm 10)$ & \\
\hline \multicolumn{7}{|l|}{ Gender } \\
\hline Male (\%) & 41.8 & 49.0 & 46.8 & 38.1 & 37.0 & 0.5 \\
\hline \multicolumn{7}{|l|}{ Smoking } \\
\hline Smoker (\%) & 14.9 & 17.5 & 24.8 & 14.3 & 18.5 & 0.3 \\
\hline Allergy to common aeroallergens (\%) & 25.4 & 34.4 & 33.3 & 43.9 & 42.3 & $<0.0001$ \\
\hline Asthma (\%) & 7.5 & 15.5 & 19.1 & 21.4 & 34.6 & $<0.05$ \\
\hline Nasal polyps (\%) & & 25.5 & 24.2 & 35.7 & 40.7 & 0.1 \\
\hline
\end{tabular}

Characteristics of the total study population given for control group (FESS $=0$ ) as well as for different surgery groups (FESS $=1 ; 2 ; 3$; and 3 + ). Smoking refers to the smoking state at the time of questionnaire. Inhalation allergy is based on either a positive skin prick test or detection of specific IgE in the blood. Asthma corresponds to self-reported asthma.

There is a significant association between number of FESS and inhalation allergy as well as between number of FESS and asthma (chi-square test). The associations between number of FESS and gender, presence of nasal polyps, or current smoking state were not significant.

The interaction of smoking, allergy, nasal polyps, and asthma in the association between occupational exposure and number of FESS was non-significant (Poisson regression model).

FESS, functional endoscopic sinus surgery; $n$, number of responders to the questionnaire in each group.

prevalent among cases than among controls; the prevalence of atopy and asthma increased significantly with increasing number of FESS (Table 1).

\section{Occupational exposures}

Among all patients undergoing FESS, 25\% reported a 'relevant' occupational exposure, which is significantly higher than the $12 \%$ found among controls (Fig. 1). The OR for reporting a relevant occupational exposure was 2.45 [95\% CI 1.14-5.29] among patients having had at least one FESS when compared to those who had no FESS.

Moreover, the proportion of patients with a relevant occupational exposure increased with the number of FESS procedures: from $21 \%$ in those operated once to $26 \%, 31 \%$, and $44 \%$ in those operated two times, three times, and more than three times, respectively $\left(\chi^{2}=12.74 ; P<0.001\right.$; Fig. 1$)$. The risk of reporting a relevant occupational exposure was significantly higher in those who needed revision sinus surgery $(\mathrm{OR}=1.63 ; 95 \%$ CI $1.06-2.51 ; P<0.05)$ when compared to those who were operated only once; the risk was even higher in those needing more than two procedures (OR $=1.97 ; 95 \%$ CI $1.14-3.42 ; P<0.05)$ when compared to those who were operated once or twice.

As indicated earlier, atopy and asthma were also more prevalent in those who had undergone more surgical procedures (Table 1). However, the relationships between occupational exposures and number of FESS procedures were not affected by adjustments for atopy, asthma, presence of nasal polyps, or smoking (data not shown).

Exposure to HMW agents was reported in $5 \%$ of the cases with relevant occupational exposures, whereas LMW sensitizers were involved in $44 \%$ and irritants in $51 \%$ of cases (Fig. 2). In $39 \%$ of the subjects reporting a relevant occupa-

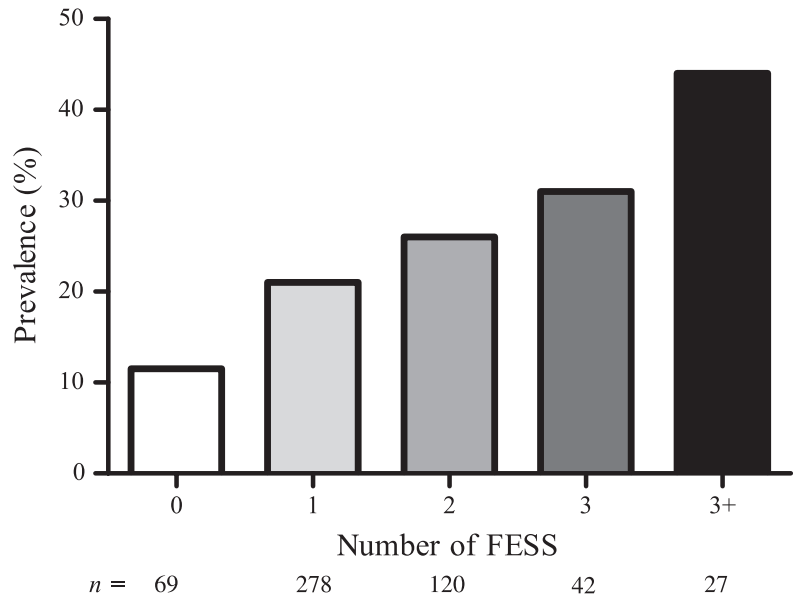

Figure 1 Prevalence of relevant occupational exposure. Prevalence of subjects reporting a relevant occupational exposure according to the number of times they underwent functional endoscopic sinus surgery (FESS). The relationship between the prevalence of subjects with relevant occupational exposure and the number of FESS was significant $\left(\chi^{2}=12.74 ; P<0.001\right.$; proportional odds model). $n=$ total number of responding subjects in each group.

tional exposure, there was coexposure to LMW allergens and irritants. The agents that were most frequently reported are listed in Table 2.

\section{Telephone survey}

Within the total study population, 110 patients were randomly selected for a control telephone survey; 62 subjects were nonresponders to the initial questionnaire and 48 were responders. 


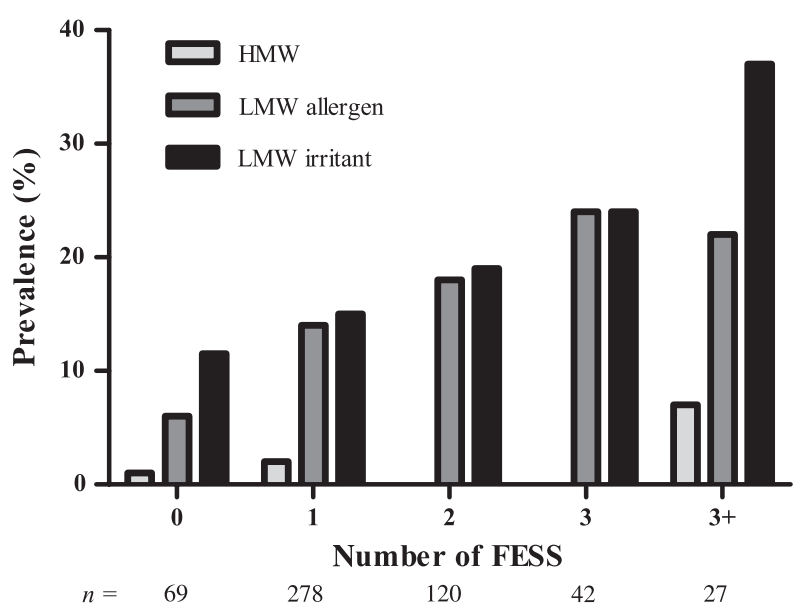

Figure 2 Prevalence of exposure to specific categories of occupational agents. Prevalence of subjects reporting occupational exposure to different categories of occupational agents according to the number of times they underwent functional endoscopic sinus surgery (FESS). HMW, high molecular weight; LMW, low molecular weight. 'LMW allergen' refers to chemicals with a known sensitizing capacity. 'LMW irritant' refers to chemicals without sensitizing capacity. Exposure to more than one category is possible. $n=$ number of responders to the questionnaire in each group.

Table 2 Most reported occupational agents

\begin{tabular}{lll}
\hline Substances & Occupation & $\begin{array}{l}\text { Times } \\
\text { reported }\end{array}$ \\
\hline Bleach & Cleaners, caretakers, housewives... & 17 \\
Inorganic dust & Builders, warehousemen, ... & 15 \\
Paints & Painters & 14 \\
Cement & Builders & 10 \\
Thinner & Painters, engineers, mechanics, ... & 10 \\
Ammonia & Carpenters, mechanics, ... & 9 \\
White spirit & Painters, engineers, mechanics, ... & 7 \\
Fuel gas & Motor/car maintenance, chauffeurs, ... & 6 \\
Acetone & Painters, mechanics, ... & 6 \\
\hline
\end{tabular}

This table lists the occupational agents that were most frequently mentioned by patients and controls. The middle column summarizes the most frequent occupations in which these agents were encountered in our study population. The right column shows the number of times the different agents were mentioned by the study subjects.

About $29 \%$ of the subjects in the responder group reported a relevant occupational exposure $v s 40 \%$ in the nonresponder group (data not shown). The results of the telephone survey and the initial written questionnaire were $83 \%$ congruent.

\section{Discussion}

We discovered that patients with rhinosinusitis who need surgery, especially repeated surgical interventions, are more likely to be exposed to inhaled noxious agents at work than a control patient population. In other words, our study reveals that occupational exposure represents a risk factor for the occurrence of rhinosinusitis and its recurrence after surgery.

It is known that airway exposure to occupational agents can give rise to occupational airway disease (16). Good epidemiologic studies investigating the prevalence of occupational rhinitis, however, are lacking. It has been postulated that occupational rhinitis occurs two to four times more often than its asthmatic counterpart $(14,17)$, and the exact prevalence is believed to be underestimated because of the current lack of validated diagnostic tools and the reluctance of patients to complain about their occupational environment for fear of losing their job.

Occupational allergic rhinitis due to HMW compounds (e.g. flour, laboratory animals,...) is easily detected by showing the presence of systemic antigen-specific $\operatorname{IgE}$ and is therefore the best-studied subgroup $(18,19)$. Thus, occupational rhinitis occurs in $18-29 \%$ of bakers and in $6-33 \%$ of people working with laboratory animals (14). However, occupational rhinitis can also be induced by sensitization to certain chemicals and other LMW compounds. Thus, 36$42 \%$ of people working with isocyanates suffer from workinduced rhinitis, and about $27 \%$ of hairdressers exposed to persulphate salts show work-related nasal symptoms (20). The detection of specific IgE against LMW chemicals is variable, and hence, it is more difficult to document sensitization. Additionally, work-related upper airway disease can be induced by exposure to irritants (e.g. chlorination products, detergents, ozone ...). Inhalation accidents with exposure to high concentrations of irritants can induce dysfunction of the upper airway mucosa, a phenomenon that has been labeled as 'reactive upper airway dysfunction syndrome' (RUDS) $(21,22)$. Several publications, however, suggest that chronic exposure to lower concentrations of irritants may lead to dysfunction of the upper respiratory tract as well $(23,24)$. In practice, these forms of occupational rhinitis are often classified among the large group of so-called idiopathic upper airway problems.

As the mucosa of the nose and sinuses forms a continuum, the sinuses are often involved in diseases that are primarily caused by inflammation of the nasal mucosa (1). Although it is still unclear whether rhinitis and rhinosinusitis share an etiological and pathophysiological background, it is highly likely that mucosal inflammation due to occupational agents may also affect the mucosa of the adjacent sinuses, leading to rhinosinusitis symptoms. Up to date, there is no description of an entity 'occupational rhinosinusitis', and the link between exposure to occupational agents and the occurrence of rhinosinusitis has never been studied so far.

Despite good success rates of medical and surgical treatment in patients with rhinosinusitis, ENT physicians still have to cope with a surgical failure percentage of about 10 $15 \%$ (1). The only proven negative outcome predictors for sinus surgery are the presence of extensive nasal polyps, cystic fibrosis, bronchial asthma, and aspirin hypersensitivity. Although final proof is needed by means of a prospective trial, our study strongly suggests for the first time that 
occupational exposure to potential harmful agents should be added to this list.

Besides the novelty of our observations, the strengths of our study include the large number of patients and the fact that our population came from a single center, thus limiting the variations in treatment strategies, surgical skills, or postoperative care. Our data confirm the known negative impacts of bronchial asthma and atopy on the prognosis of FESS $(12,25,26)$, showing the relevance of our study population. We remark that the percentage of patients who only need one surgery to control symptoms is lower in this study population $(59.5 \%)$ compared to the general population (85-90\%) (1). This can be explained by the fact that our department is a tertiary referral center in which a high number of revision surgeries are performed.

Our study also has some limitations. One limitation relates to our assessment of exposures to occupational agents. Occupational exposures were derived from the replies to a selfadministered questionnaire. A possible concern is that the response rate to the questionnaire could be nondifferential, meaning that patients with significant occupational exposures might have been more prone to reply to the questionnaire, because of a tendency to attribute their condition to dirty work. However, a telephone survey in a random $10 \%$ sample of our subjects did not show a higher response rate to the postal questionnaires among exposed subjects than among nonexposed subjects. Another limitation might be our assessment of 'relevance' of the reported occupational exposures. This relevance was verified by three physicians with experience in occupational health, who judged blindly and independently from each other whether the reported exposures were substantial enough to have an impact on their airways. Exposure was considered relevant only if two of the three experts had rated the exposure as positive. Alternative methods, such as specific job-exposure matrices (JEM), exist for the retrospective assessment of occupational exposures, but JEMs are not necessarily more valid, and expert judgment based on self-reported job descriptions has been used in many studies of occupational exposures and proven to be reliable $(27,28)$.

We did not simply compare patients who had undergone sinus surgery with patients who had not, but we also compared subjects who underwent multiple FESS with those who had only one FESS. The design of our study was appropriate to test our hypothesis on exposure to occupational agents in relation to a need for revision sinus surgery. Here, the prevalence of reported work exposures among those who had more than one FESS was higher than among those with only one FESS. Moreover, the increase in the prevalence of occupational exposures with increasing number of FESS procedures provides strong support for a causal relationship between work exposure and the failure of FESS. Importantly, our results were not due to confounding factors like atopy, concomitant asthma, nasal polyps, or current smoking.

Although HMW agents such as flour, latex, and laboratory animals are the best-known causal factors of occupational airway disease (29), we found here that exposure to such classical inducers of IgE-mediated allergic asthma and rhinitis was not so prevalent $(<5 \%)$ and the results were mainly driven by LMW agents. LMW sensitizers as well as irritants were frequently reported and often there was coexposure to both groups.

Elucidating the pathogenesis of occupational rhinosinusitis and why LMW agents contribute to failure of healing following FESS is beyond the scope of this observational study.

In conclusion, we provide novel evidence for exposure to occupational agents influencing the outcomes of sinus surgery and playing a role in the development and maintenance of sinonasal disease. The clinical implication of these findings is that exposures at work should be taken into account when evaluating and treating patients with rhinosinusitis. Moreover, apart from pointing to a continued need of prevention of harmful exposures at work, our results also emphasize the need for further research in this area, by means of prospective clinical studies as well as through fundamental research exploring pathophysiological mechanisms of occupational upper airway disease.

\section{Acknowledgments}

The project was supported by a grant of the Interuniversity Attraction Pole Program, Belgian State, Belgian Science Policy P6/35, and from the 'Fonds voor Wetenschappelijk Onderzoek Vlaanderen' (FWO): P. H. and J.V. are postdoctoral research fellows, and V. H. is a research fellow of the FWO.

\section{Authors' contributions}

Valérie Hox*: study design, questionnaire design, data collection, data analysis, analysis of questionnaires, telephone survey, literature search, writing manuscript. Stefan Delrue*: patient recruitment, data collection, data analysis, telephone survey, literature search, writing manuscript. Hans Scheers: data analysis, statistical analysis, writing manuscript. Els Adams: analysis of questionnaires. Stephan Keirsbilck: analysis of questionnaires. Mark Jorissen: surgeon, data interpretation, correcting manuscript. Peter Hoet: data interpretation, correcting manuscript. Jeroen A. Vanoirbeek: data interpretation, correcting manuscript. Benoit Nemery: study design, data interpretation, statistical analysis, writing manuscript. Peter W. Hellings: study design, surgeon, data interpretation, writing manuscript. *These authors contributed equally.

\section{Funding}

The project was supported by a grant of the Interuniversity Attraction Pole Program, Belgian State, Belgian Science Policy P6/35, and from the 'Fonds voor Wetenschappelijk Onderzoek Vlaanderen' (FWO): P. H. and J.V. are postdoctoral research fellows, and V. H. is a research fellow of the FWO.

\section{Conflict of interest}

All authors declare that there are no conflicts of interest. 


\section{References}

1. Fokkens W, Lund V, Mullol J. EP3OS 2007: European position paper on rhinosinusitis and nasal polyps 2007. A summary for otorhinolaryngologists. Rhinology 2007;45:97 -101 .

2. Emanuel IA, Shah SB. Chronic rhinosinusitis: allergy and sinus computed tomography relationships. Otolaryngol Head Neck Surg 2000; 123:687-691.

3. Krause HF. Allergy and chronic rhinosinusitis. Otolaryngol Head Neck Surg 2003;128:14-16.

4. Bhattacharyya $\mathrm{N}$. The role of infection in chronic rhinosinusitis. Curr Allergy Asthma Rep 2002;2:500-506.

5. Chee L, Graham SM, Carothers DG, Ballas ZK. Immune dysfunction in refractory sinusitis in a tertiary care setting. Laryngoscope 2001;111:233-235.

6. Jones NS. CT of the paranasal sinuses: a review of the correlation with clinical, surgical and histopathological findings. Clin Otolaryngol Allied Sci 2002;27: 11-17.

7. Wang X, Moylan B, Leopold DA, Kim J, Rubenstein RC, Togias A et al. Mutation in the gene responsible for cystic fibrosis and predisposition to chronic rhinosinusitis in the general population. JAMA 2000;284:1814-1819.

8. King JM, Caldarelli DD, Pigato JB. A review of revision functional endoscopic sinus surgery. Laryngoscope 1994;104:404 408.

9. Marks SC, Kissner D. Endoscopic sinus surgery in patients with cystic fibrosis. Otolaryngol Head Neck Surg 1996;114:840-841.

10. Marks SC, Shamsa F. Evaluation of prognostic factors in endoscopic sinus surgery. Am J Rhinol 1997;11:187-191.

11. McMains KC, Kountakis SE. Revision functional endoscopic sinus surgery: objective and subjective surgical outcomes. Am J Rhinol 2005;19:344-347.
12. Lavigne F, Nguyen CT, Cameron L, Hamid Q, Renzi PM. Prognosis and prediction of response to surgery in allergic patients with chronic sinusitis. J Allergy Clin Immunol 2000a;105:746-751.

13. Young J, Frenkiel S, Tewfik MA, Mouadeb DA. Long-term outcome analysis of endoscopic sinus surgery for chronic sinusitis. Am J Rhinol 2007;21:743-747.

14. Moscato G, Vandenplas O, Van Wijk RG, Malo JL, Perfetti L, Quirce S et al. EAACI position paper on occupational rhinitis. Respir Res 2009;10:16.

15. Bernstein I, Chan-Yeung M, Malo JL, Bernstein D. Occupational history form. In: Bernstein I, Chan-Yeung M, Malo JL, Bernstein DI, editors. Asthma in the Workplace. New York, NY: Marcel Dekker, 1999: 731734.

16. Bousquet J, Van CP, Khaltaev N. Allergic rhinitis and its impact on asthma. J Allergy Clin Immunol 2001;5Suppl:S147-S334.

17. Moscato G, Vandenplas O, Gerth Van WR, Malo JL, Quirce S, Walusiak J et al. Occupational rhinitis. Allergy 2008;63:969-980.

18. Rodier F, Gautrin D, Ghezzo H, Malo JL. Incidence of occupational rhinoconjunctivitis and risk factors in animal-health apprentices. J Allergy Clin Immunol 2003;112:1105-1111.

19. Storaas T, Steinsvag SK, Florvaag E, Irgens A, Aasen TB. Occupational rhinitis: diagnostic criteria, relation to lower airway symptoms and IgE sensitization in bakery workers. Acta Otolaryngol 2005;125:12111217.

20. Moscato G, Pignatti P, Yacoub MR, Romano C, Spezia S, Perfetti L. Occupational asthma and occupational rhinitis in hairdressers. Chest 2005;128:3590-3598.

21. Hoffman CD, Henneberger PK, Olin AC, Mehta A, Toren K. Exposure to ozone gases in pulp mills and the onset of rhinitis. Scand $J$ Work Environ Health 2004;30:445-449.
22. Meggs WJ, Elsheik T, Metzger WJ, Albernaz M, Bloch RM. Nasal pathology and ultrastructure in patients with chronic airway inflammation (RADS and RUDS) following an irritant exposure. J Toxicol Clin Toxicol 1996;34:383-396.

23. Bougult V, Turmel J, Boulet LP. Effect of intense swimming training on rhinitis in high-level competitive swimmers. Clin Exp Allergy 2010;40:1238-1246.

24. de Fatima ME, Algranti E, Medina Coeli ME, Antonio BM. Rhinitis and asthma symptoms in non-domestic cleaners from the Sao Paulo metropolitan area, Brazil. Occup Environ Med 2007;64:446-453.

25. Li Y, Zhang GH, Liu X, Ye J, Zeng ZP. Clinical prognostic factors of chronic rhinosinusitis after endoscopic sinus surgery. $O R L$ J Otorhinolaryngol Relat Spec 2008;70:113117.

26. Smith TL, Mendolia-Loffredo S, Loehrl TA, Sparapani R, Laud PW, Nattinger AB. Predictive factors and outcomes in endoscopic sinus surgery for chronic rhinosinusitis. Laryngoscope 2005;115:2199-2205.

27. Peters S, Vermeulen R, Cassidy A, Mannetje A, van TM, Boffetta P et al. Comparison of exposure assessment methods for occupational carcinogens in a multi-centre lung cancer case-control study. Occup Environ Med 2011;68:148-153.

28. Quinlan PJ, Earnest G, Eisner MD, Yelin EH, Katz PP, Balmes JR et al. Performance of self-reported occupational exposure compared to a job-exposure matrix approach in asthma and chronic rhinitis. Occup Environ Med 2009;66:154-160.

29. Siracusa A, Desrosiers M, Marabini A. Epidemiology of occupational rhinitis: prevalence, aetiology and determinants. Clin Exp Allergy 2000;30:1519-1534. 